\title{
Multi-Scale Stochastic Optimization for Home Energy Management
}

\author{
Liyan Jia, Zhe Yu, Mary C. Murphy-Hoye, Annabelle Pratt, Ellen G. Piccioli, and Lang Tong
}

\begin{abstract}
The problem of scheduling and control of appliances for Home Energy Management (HEM) is considered. A multitime scale and multi-stage stochastic optimization framework is proposed for the control of the Heating, Ventilation, and Air Conditioning (HVAC) unit, the charging of Plug-in Hybrid Electric Vehicle (PHEV), and the scheduling of deferrable load such as washer/dryer operations. Formulated as a constrained stochastic optimization that incorporates thermal dynamics, temperature measurements, and the real time pricing signal, a model predictive control algorithm is proposed that minimizes customer's discomfort level subject to cost and peak power constraints.
\end{abstract}

Index Terms-Home energy management, model predictive control, smart grid, demand response, HVAC control, PHEV charging.

\section{INTRODUCTION}

W E consider the problem of optimal control and scheduling of appliances by a Home Energy Management (HEM) device. The basic premise of this work is that the HEM device at the customer site serves as a gateway in the interaction with either the utility or an energy aggregator. In the context of a hierarchical demand side management (DSM) system, for example, an energy aggregator interfaces with a Retail Electric Provider (REP) and a pool of customers. In a hypothetical DSM operation, as illustrated in Fig. 1, the aggregator secures a contract with the REP and promises an aggregated load profile among its customers. The aggregator incentivizes its customers by a certain pricing scheme to fulfill the promised load profile. The result is that each customer provides the aggregator with its own load profile that specifies the maximum power consumption. In the event of required load shifting, the aggregator may send requests of changes in individual load profiles, and the HEM device of the customer can respond accordingly.

An essential function of an HEM device is to manage energy consumption based on the load profile promised to the aggregator, the pricing signal from the aggregator, the sensing devices that measure the home environment, the desired comfort level, and budget constraints. The customer may specify a certain monthly energy expenditure and expect the HEM device to optimize intelligently energy usage that fits the customer's specific lifestyle.

L. Jia, Z. Yu, and L. Tong are with the School of Electrical and Computer Engineering, Cornell University, Ithaca, NY 14853, USA. Email: $\{1 j 92$, zy73, lt35\} ecornell.edu. M. Murphy-Hoye, A. Pratt, and E. Piccioli are with Intel Corp., USA.

This work is supported in part by the Intel Fellowship Program and the National Science Foundation under CNS 1135844.

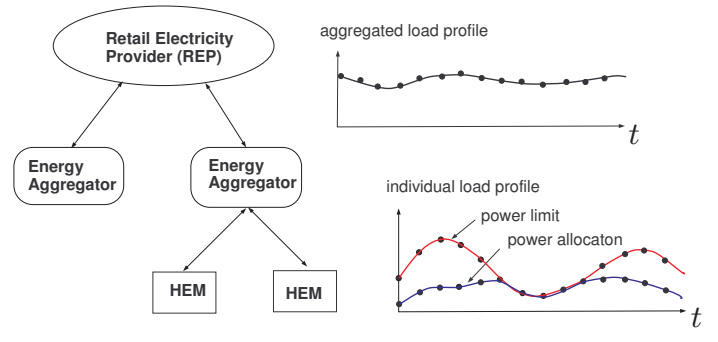

Fig. 1: A hierarchical demand side management system.

\section{A. Summary of Results}

The main contribution of this paper is a computationally tractable multi-scale multi-stage optimization framework that integrates various functionalities of HEM. The multi-scale and multi-stage nature of the problem arises naturally in home energy management. For example, the thermal dynamics of a residential home may be modeled the minute level, the load profile for the maximum power consumption is specified at the hourly level, and the targeted energy expenditure is given at weekly or monthly level. The scheduling of various devices also involves multiple stages as information required for scheduling arrives at different time, which affects the time when scheduling decisions are made. Unfortunately, finding the optimal scheduling policy of a multi-scale multi-stage stochastic program is intractable in general.

We propose a hierarchical approach that separates the problem into slow and fast scale optimizations; the former provides the hourly power (energy) budget for different devices whereas the latter determines control signals at the time scale that matches to that of the thermal dynamics (minute level). The proposed approach also separates continuous variable from integer variable optimizations.

To provide power allocation at the slow time scale, the optimization involves a quadratic optimization with linear constraints, which can be solved easily using standard numerical techniques. At the fast time scale, because of the on-off nature of HVAC control, the problem becomes a stochastic integer program where a suboptimal heuristic approach is proposed.

The underlying principle for the multi-stage stochastic optimizations at the two different time scale is the Model Predictive Control (MPC) [1]. In particular, MPC forecasts the state of the physical plant into the future and makes tentative future decisions optimally based on such forecasts. The key of 
MPC is to excersize only the immediate tentative decision and refine its forecast as it collects more information. MPC does not lead to the optimal solution in general, but the strategy is widely used in practice; it is computationally tractable and often offers satisfactory performance.

\section{B. Related Work}

The literature on home energy management is expanding rapidly, but few published work provides an integrated approach to loads of different types, addressing design tradeoffs among comfort requirements and peak power and budget constraints. Authors of [2] proposed a three-layer control mechanism and use Tabu search to find a feasible solution. In [3], particle swarm optimization is used to find the optimal solution for coordinately scheduling multiple energy resources. These approaches require accurate prediction of the energy usage of future. In [4], uncertainty consideration is incorporated into the optimization, but the control is an open loop strategy without using the real-time measurement.The authors of [5] considered a similar scheduling problem as one treated in this paper. The emphasis in [5] is on the tradeoff between cost and waiting time in a multi-home setting. The work presented here, in contrast, focuses on energy management problem within a single home with a design tradeoff between cost and comfort level subject to budget and power constraints. It is also significant that the scheduling problem considered in this paper involves thermal dynamics that dictates the formulation of multi-stage stochastic dynamic optimization. The thermal dynamics is not modeled in [5] and the optimization involved is considerably simpler.

The MPC strategy adopted in this paper goes back to [6], [7], [8]. In [6], an algorithm referred to as LQG-MPC was proposed to deal with the state and control linear inequality constraints. In [7], [8], the Quadratic Dynamic Matrix Control is used to solve nonlinear process optimization with state estimation.

\section{Problem Formulation And HEM Architecture}

We present in this section the basic system model and the overall solution architecture. In dealing with models in multiple time scale, we adopt the notation that $x[t]$ stands for the representation of signal in the fast time scale (say in minutes) whereas $x_{n}$ represents the signal in the slow time scale (e.g., for hourly measurements).

\section{A. Load classes and characteristics}

We consider three types of load in this paper: The first is the HVAC unit which draws power $p^{\mathrm{HVAC}}[t]$ and drives the indoor room temperature $x^{\text {in }}[t]$ following a thermal dynamic model given in Sec. II-B. The control of HVAC directly affects the quality of service and is subject to peak power and budget constraints. The quality of service is defined by the level of discomfort measured by the deviation of room temperature from the desired setting.

The second type of load is the charging of PHEV. This is a deferrable and interruptible load. By preemption we mean that the charging of the PHEV can be suspended temporarily and resumed at a later time. We assume that the charging can start at $T_{A}$ and must be completed by $T_{D}$. The control strategy affects the comfort level of the customer indirectly through the constraints on peak power and available budget. We assume in this paper that the PHEV must charged to a certain level by the deadline, putting this job at a higher priority over minimizing the discomfort level.

The third load type is deferrable and non-interruptible load. The example considered in this paper is the scheduling of washer or dryer for which the the start time of the load can be moved based on power consumption and pricing information. Here the control is the start time of the load within the earliest start time $T_{E}$ and the latest start time $T_{L}$. The load characteristics (power drawn from the grid) is assumed to be known. As in the control of PHEV charging, the schedule of deferrable load is a hard requirement and it affects the level of comfort indirectly.

\section{B. MIMO Thermal Dynamics}

We assume a multi-input and multi-output (MIMO) model that specifies the indoor room temperature evolution $x^{\text {in }}[t]$ as a function of the outdoor temperature $x^{\text {out }}[t]$ and the power of multiple HVACs $p^{\mathrm{HVAC}}[t]$. As a generalization of the standard dynamic model for residential air conditioning, the model is a stochastic linear difference equation given by

$$
\begin{aligned}
\Phi(A, G, C): \quad & x[t+1]=A x[t]+G p^{\mathrm{HVAC}}[t]+v[t] \\
& y[t]=C x[t]+w[t]
\end{aligned}
$$

where the state vector $x[t] \triangleq\left(x^{\text {in }}[t], x^{\text {out }}[t]\right)$ consists of the indoor temperature $x_{t}^{\text {in }}$ and outdoor temperature $x^{\text {out }}[t]$. The multiple HVACs are controlled via vector $p^{\mathrm{HVAC}}[t]$. The measurement is denoted as $y[t]$. The model includes process noise $v[t]$ and measurement noise $w[t]$, both assumed to be zeromean white Gaussian noise with known covariances.

We have performed validation of the above model using real data collected from a residential home. The measurements include indoor, outdoor temperature, and HVAC power usage. Model parameters are extracted by the least squares method using one month of measurements. The validation of the model is performed using data in two different months. The modeling and prediction mean squared error are below 0.1 degree [9].

\section{Control Policy, Cost, and Figures of Merits}

A control/scheduling policy $\pi$ is a power allocation to the three types of loads $\left(p_{\mathrm{HVAC}}^{\pi}[t], p_{\mathrm{PHEV}}^{\pi}[t], p_{\mathrm{Def}}^{\pi}[t]\right)$ at the fast time scale (minute intervals) using measurement $\mathscr{Y}_{[0: t)}$ up to $t$.

Let $\mathscr{P}$ be the set of admissible policies that satisfy the scheduling constraints including the required start-end time for charging and start time for deferrable loads. Of particular importance is the the vector of aggregated hourly load constraints $\left(P_{1}, \cdots, P_{T_{h}}\right)$. Specifically, given a control policy $\pi \in \mathscr{P}$, let $p^{\pi}[t] \triangleq p_{\mathrm{HVAC}}^{\pi}[t]+p_{\mathrm{PHEV}}^{\pi}[t]+p_{\text {Def }}^{\pi}[t]$ be the aggregated power consumption in interval $t$. The hourly power consumption 
limits mandates that

$$
p_{i}^{\pi}=\sum_{t \text { in hour } i} p^{\pi}[t] \leq P_{i}, \quad i=0,1, \cdots, T_{h} .
$$

Given the price signal $z[t]$, the cost of a policy $\pi$ is give by

$$
\mathcal{C}(\pi) \triangleq \sum_{t} z[t] \times p^{\pi}[t] .
$$

We measure the (lack of) quality of a policy $\pi \in \mathscr{P}$ by the discomfort level by

$$
\mathcal{D}(\pi) \triangleq \mathbb{E}_{\pi}\left(\sum_{t}\left\|x^{\mathrm{in}}[t]-d[t]\right\|^{2}\right),
$$

where $x^{\text {in }}[t]$ is the indoor temperature and $d[t]$ the desired temperature settings. Given a daily budget constraint $B$, the optimal policy is the solution of the following constrained optimization

$$
\min _{\pi \in \mathscr{P}} \mathcal{D}(\pi) \quad \text { subject to } \quad \mathcal{C}(\pi) \leq B .
$$

\section{HEM Control Architecture}

We propose a multi-scale and multi-stage control architecture shown in Fig. 2 with detailed functionalities of the slow and fast time scale optimizations defined in Sections III-IV.

The architecture is based on the principle of model predictive control (MPC). Sensor measurements (indoor and outdoor temperatures) $y[t]$ are taken at the fast time scale and a Kalman filter is used to predict future thermal dynamic states $\hat{x}[t+k \mid t]$. The slow time scale MPC uses state prediction and pricing signal $z[t]$ to allocate power budget to the three types of load at the slow time scale, and the fast time scale MPC determines the detailed control at the fast time scale.

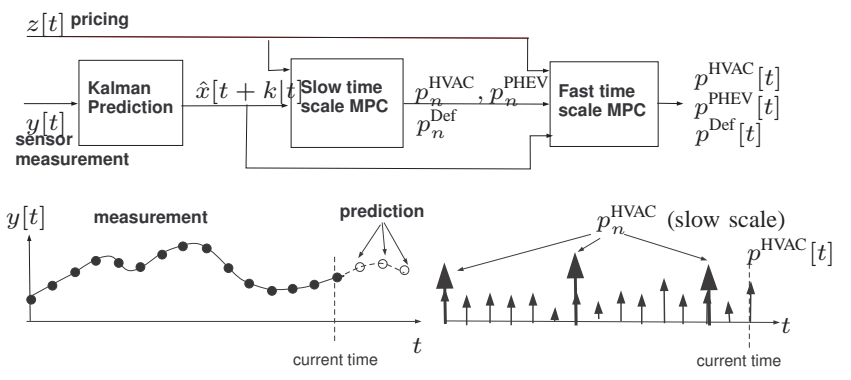

Fig. 2: Multi-scale HEM architecture.

\section{Slow Time Scale Stochastic Optimization}

A control policy $\pi$ is a mapping from measurements $\mathscr{Y}_{0, n}=$ $\left\{y_{n}, y_{n-1}, \cdots, y_{0}\right\}$ to decision variables $\left(p_{n}^{\mathrm{HVAC}}, p_{n}^{\mathrm{PHEV}}, s\right)$ where $s$ is the start time of the deferrable load. Note that, once the start time is determined, the power allocation to the deferrable load at the fast time scale is determined.

The goal of the slow time scale optimization is to minimize the discomfort level subject to power limit and budget constraint. To this end, we consider the following multi-stage stochastic optimization:

$$
\begin{aligned}
& \operatorname{minimize} \quad \sum_{t=0}^{T_{h}} \mathbb{E}_{\pi}\left\|x_{t}^{\text {in }}-d_{t}\right\|^{2} \\
& \text { subject to }\left(x_{t}, y_{t}\right) \sim \Phi(A, G, C) \\
& T_{E} \leq s \leq T_{L} \\
& p_{t}^{\text {Def }}= \begin{cases}P_{\text {rate }}^{\text {Def }} & \text { if } s \leq t<s+d \\
0 & \text { o.w. }\end{cases} \\
& 0 \leq p_{t}^{\text {PHEV }} \leq P_{\text {max }}^{\text {PHEV }} \\
& \sum_{t=0}^{t=T_{h}-1} p_{t}^{\text {PHEV }}=Q \\
& p_{t}^{\text {PHEV }}=0 \quad \text { if } t<T_{A} \text { or } t>T_{D} \\
& 0 \leq p_{t}^{\mathrm{HVAC}}+p_{t}^{\mathrm{PHEV}}+p_{t}^{\mathrm{Def}} \leq P_{t} \\
& \sum_{t=0}^{T_{h}-1} z_{t}\left(p_{t}^{\mathrm{HVAC}}+p_{t}^{\mathrm{PHEV}}+p_{t}^{\mathrm{Def}}\right) \leq B
\end{aligned}
$$

where $x_{t}^{\text {in }}$, the indoor temperature vector, is part of the state evolution $\left(x_{t}, y_{t}\right) \sim \Phi(A, G, C)$ specified by the stochastic thermal dynamic equation (1). For simplicity, we will assume that the deferrable load draws constant rated power $P_{\text {rate }}^{\text {Def }}$.

\section{Fast Time Scale Stochastic Optimization}

We now consider the scheduling and control at the fast time scale. The principle established for the slow time scale optimization applies here as well, except that we now deal with scheduling with integer decision variables.

Due to space limitation, we focus on the control of HVAC at the fast time scale. The most widely used control strategy is the on-off control of HVAC where the heating and air conditioning is turned on or off based on the desired temperature set point and actual temperature measurements [10]. In practice, considerations of equipment longevity may put additional constraints on how frequent the switching between different states can be.

Within the class of on-off controls, we formulate the problem as choosing the on-off switching time subject to minimize the discomfort level and subject to the hourly power budget constraint. Once the switching time is chosen, the control of HVAC can be implemented through artificially choosing the desired temperature set points without actually install a different controller.

The fast time scale control of HVAC starts by calculating the number of on periods within each hour, assuming that the HVAC uses the same power level when it is turned on and schedule the on periods by a stochastic optimization. In particular, given the total power budget $p_{k}^{\mathrm{HVAC}}$ in the $k$ th hour, the number of on periods is given by $N_{k}=\left[p_{k}^{\mathrm{HVAC}} / P_{\text {rate }}^{\mathrm{HVAC}}\right]$. The allocation of the on periods is formulated as a stochastic optimization with the binary action space.

\section{Simulations}

We present preliminary simulations to illustrate the proposed approach. Three types of loads are included in the simulation. As a deferrable load, the dryer is assumed to last for two hours, assumed starting no earlier than 4PM and completing no later than 6PM. We also assume that the PHEV is available for charging between $8 \mathrm{PM}$ and $8 \mathrm{AM}$. The charing 
rate is nonnegative which implies it cannot discharge. A peak power constraint is applied by the HEM as well as a total budget constraint.

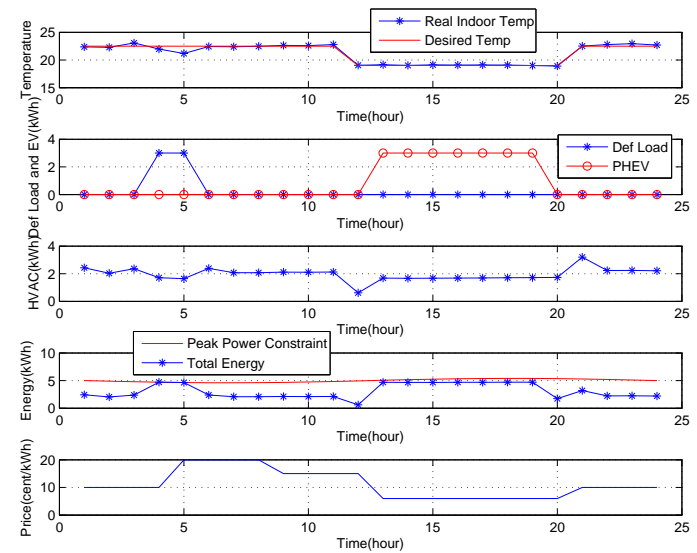

Fig. 3: Power Policy Example: Indoor Temp, Dryer and PHEV Charing, HVAC, Total Energy and Peak constraints, Price

Fig. 3 illustrates on resulting control policy. Given the tight schedule deadline for the deferrable load, the optimal scheduling for the dryer was between 4PM-6PM. Note also that the PHEV charging schedule took advantage the price advantage. Given the consumption limit, there are several periods that the power limits became binding. As a result, there were two hourly intervals that less power allocation to the HVAC were made, which led to deviation from the desired temperature.

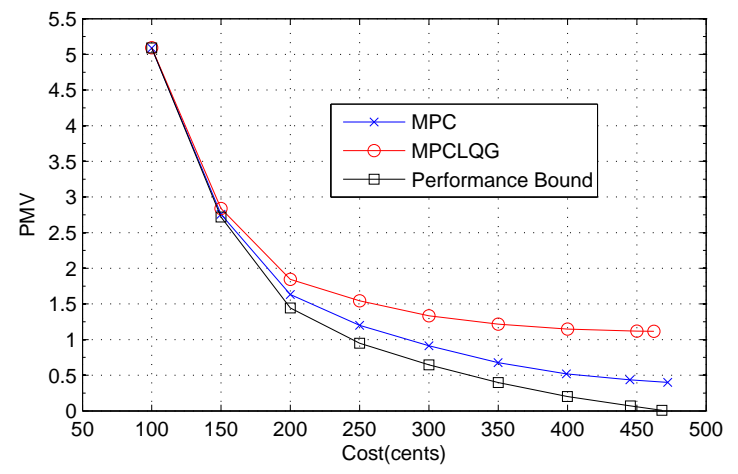

Fig. 4: Performance comparison between LQG-MPC and Multi-scale Algo

We conducted a comparison between the proposed approach and a benchmark solution. Given that there is no existing approach to HEM that involves different types of services considered here, we focused on the scheduling of HVAC under peak power and budget constraints. In particular, we compared with an LQG-based MPC strategy (thus referred to as LQGMPC) originally proposed in [6], which can be viewed as an interpolation between LQG and an open loop solution.
The performance bound is obtained by knowing the future perfectly.

The plot of discomfort level (measured in terms of Predicted Mean Value(PMV)) against energy cost is shown in Fig. 4 where the proposed approach performed better than LQGMPC. At the PMV value of 1.5, the saving of the proposed approach over LQG-MPC was about 20\%. While the MPC spend $8 \%$ more than the optimal condition. We noted that, when the budget constraint was tight, the difference between the three approaches was small, which can be explained by the fact that both strategies have limitted power available for scheduling.

\section{CONCLUSION}

We presented in this paper a multi-scale multi-stage stochastic optimization framework for home energy management that involves loads with different characteristics. With HEM device as an interface with the energy aggregator through real-time pricing and economically incentivized load profile, our goal here is to provide a conceptual decomposition of the optimization problem into computationally tractable subproblems.

We have made a number of simplifying assumptions that need to be justified and studied further for practical implementations. The linear thermal model needs to be validated, and the assumption that model parameters are known needs to be replaced by online parameter estimation and tracking algorithms. The impact of the economically based scheduling on the life-time of appliances, especially in the context of PHEV charging and bang-bang HVAC control, warrants further study. Nonetheless, many of the modifications required to circumvent the above simplifying assumptions can be incorporated into the proposed optimization framework.

\section{REFERENCES}

[1] J. Rawlings, "Tutorial overview of model predictive control," IEEE Control Systems Magazine, June 2000.

[2] D. Ha, S. Ploix, E. Zamai, and M. Jacomino, "Tabu search for the optimization of household energy consumption," in 2006 IEEE Int. Conf. Inf. Reuse Integr., pp. 86 - 92, 2006.

[3] M. Pedrasa, T. Spooner, and I. MacGill, "Coordinated Scheduling of Residential Distributed Energy Resources to Optimize Smart Home Energy Services," IEEE Trans. on Smart Grid, vol. 1, September 2010.

[4] D. L. Ha, M. H. Le, and S. Ploix, "An approach for home load energy management problem in uncertain context," in Industrial Engineering and Engineering Management, 2008. IEEM 2008. IEEE International Conference on, pp. $336-339$, dec. 2008.

[5] A. Mohsenian-Rad and A. Leon-Garcia, "Optmal Residential Load Control With Price Prediction in Real-Time Electricity Pricing Environments," IEEE Tran. On Smart Grid, vol. 1, pp. 120-133, Sept 2010.

[6] M. Sznaier and M. J. Damborg, "Suboptimal control of linear systems with state and control inequality constraints," in Decision and Control, 1987. 26th IEEE Conference on, vol. 26, pp. 761 -762, dec. 1987.

[7] N. L. Ricker, "Model predictive control with state estimation," Ind. Eng. Chem. Res., vol. 29, no. 3, pp. 374-382, 1990.

[8] G. Gattu and E. Zafiriou, "Nonlinear quadratic dynamic matrix control with state estimation," Industrial and Engineering Chemistry Research, vol. 31, no. 4, pp. 1096-1104, 1992.

[9] L. McLaughlin, L. Jia, Z. Yu, and L. Tong, "Thermal Dynamic for Home Energy Management: A case study," Tech. Rep. ACSP-TR-10-11-01, Cornell University.

[10] B. Li and A. Alleyne, "Optimal on-off control of an air conditioning and refrigeration system," in American Control Conference (ACC), 2010, pp. $5892-5897$, July 2010. 